\title{
MODIFIED INTEGRATION ALGORITHM IN THE STRAIN SPACE FOR RATE AND TEMPERATURE DEPENDENT ELASTO-PLASTIC CONSTITUTIVE MODEL
}

\author{
SANG-SOON $\mathrm{CHO}^{1, \mathrm{a}}$ AND HOON HUH ${ }^{1, \mathrm{~b}}$ \\ ${ }^{1}$ Department of Mechanical Engineering, Korea Advanced Institute of Science Technolgy,373-1, \\ Science Town, Daejeon, 305-701 Korea \\ ass211003@kaist.ac.kr, ${ }^{b} h h u h @ k a i s t . a c . k r$ \\ Received Day Month Day \\ Revised Day Month Day
}

\begin{abstract}
This paper is concerned with modified integration algorithm in the strain space for rate and temperature dependent elasto-plastic constitutive relations in order to obtain more accurate results in numerical implementation. The proposed algorithm adopts both the 2-stage Lobatto IIIA with second-order accuracy and the iteration procedure using the Newton-Raphson method to enfoce consistency at the end of the step. The algorithm enables to consider the convective stress rates on the yield surface in the strain space in the numerical integration of the constitutive model. Accuracy assessment using the isoerror maps and FEM analysis of the adiabatic shear band is carried out in order to demonstrate the accuracy of the proposed algorithm.
\end{abstract}

Keywords: Backward-Euler return method; 2-stage Lobatto IIIA; Rate and Temperature Dependent Constitutive Model; Isoerror maps; Adiabatic shear bands

\section{Introduction}

Strain-rate and temperature dependent constitutive models are widely utilized in the numerical simulation for materials under dynamic loading conditions. Since most materials show their inherent rate and temperature dependent behaviors in various strainrate. Numerical implementations for the constitutive equation such as the Johnson-Cook model are typically carried out on the basis of theoretical results which are derived on the stress space formulation. However the stress space formulation in rate independent plasticity possesses the singularity in the constitutive equation and cannot distinguish between the plastic loading and elastic unloading for work-softening materials. Therefore it has the possibility of confusion in loading-unloading criteria when the plastic strainrates are fluctuated or thermal softening takes place. The strain space formulation proposed by Il'yushin is attractive alternatives to eliminates confusion in the definition of the loading-unloading criterion and also provide a unified treatment for work-hardening or softening materials. ${ }^{1}$

The backward-Euler return algorithm is the most widely used integration procedure for the classical $\mathrm{J}_{2}$ elasto-plasticity. This scheme consists of elastic predictor-plastic corrector algorithm where a purely elastic trial state is followed by a plastic corrector 
phase. But this method cannot consider the histories of the stress rates on the yield surface during the plastic deformation since it just guarantees first-order accuracy. ${ }^{2}$

This paper is concerned with modified integration algorithm in the strain space for rate and temperature dependent elasto-plastic constitutive relations in order to obtain more accurate results in numerical implementation. The proposed algorithm is integrated analytically using integration by part and chain rule and then is applied to the 2-stage Lobatto IIIA with second-order accuracy. ${ }^{3}$ The suggested algorithm enables to consider the convective stress rates on the yield surface of the strain space. The accuracy of the proposed algorithm is assessed by isoerror maps for rate independent constitutive relation. Analysis of the adiabatic shear bands is also carried out in order to demonstrate the accuracy of the proposed algorithm for rate and temperature dependent constitutive relation.

\section{Numerical Integration Formulation based on the Strain Space}

\subsection{Strain space plasticity for rate and temperature dependent constitutive model}

For rate and temperature dependent constitutive model, the von Mises yield criterion in the strain space can be expressed as follows; ${ }^{1}$

$$
\varphi\left(\boldsymbol{\varepsilon}^{\prime e}, \varepsilon^{p}, d^{p}, T\right)=\sqrt{6 \mu^{2}\left(\boldsymbol{\varepsilon}^{\prime}-\boldsymbol{\varepsilon}^{p}\right):\left(\boldsymbol{\varepsilon}^{\prime}-\boldsymbol{\varepsilon}^{p}\right)}-g\left(\varepsilon^{p}, d^{p}, T\right)=0 .
$$

, where the function $g\left(\varepsilon^{p}, d^{p}, T\right)$ is the yield function in the strain space and $\varepsilon^{p}, d^{p}$ and $T$ mean effective plastic strain, effective plastic strain rate and temperature respectively. In conjunction with Eq.(1), the Prandtl-Reuss flow rule can be expressed as Eq.(2)

$$
\mathbf{D}^{p}=\frac{1}{2 \mu} d^{p} \frac{\partial \varphi}{\partial \boldsymbol{\varepsilon}}=\frac{1}{2 \mu} d^{p} \mathbf{a} .
$$

, where $\mathbf{D}^{p}$ is the plastic deformation rate and the vector $\mathbf{a}$ is normal to the yield surface. Elastic deformation rate can be written as shown in Eq.(3) by the additive decomposition of the deformation rate. ${ }^{1}$

$$
\mathbf{D}^{e}=\mathbf{D}-\mathbf{D}^{p}=\mathbf{D}-\frac{1}{2 \mu} d^{p} \frac{\partial \varphi}{\partial \boldsymbol{\varepsilon}}
$$

According to the consistency condition and chain rule, the yield function of Eq.(1) should satisfy

$$
d \varphi=\frac{\partial \varphi}{\partial \boldsymbol{\varepsilon}} d \boldsymbol{\varepsilon}^{e}-\left(\frac{\partial g}{\partial \varepsilon^{p}} d \varepsilon^{p}+\frac{\partial g}{\partial d^{p}} d\left(d^{p}\right)+\frac{\partial g}{\partial T} d T\right)=0 .
$$

This paper deals with adiabatic shear deformation which describes the localization of plastic flow at high strain rate to large plastic strain. Therefore temperature change can be expressed as follows;

$$
\dot{T}=\chi \frac{\sigma_{e f f} d^{p l}}{\rho c_{p}} .
$$


, where $\rho$ is the density of the material, $c_{p}$ is the heat capacity of the material and $\chi$ defines the fraction of plastic. With these Eq.(1) (5) the effective plastic strain rate is written as shown in Eq.(6).

$$
d^{p}=\frac{\mathbf{a}^{T} \mathbf{D}}{3 \mu+\left(\frac{\partial g}{\partial \varepsilon^{p}}+\frac{1}{\Delta t} \frac{\partial g}{\partial d^{p}}+\frac{\partial g}{\partial T} \frac{\chi \bar{\sigma}}{\rho c_{p}}\right)}=\frac{\mathbf{a}^{T} \mathbf{D}}{3 \mu+H}
$$

where $H$ means the slope of stress-strain curve of rate and temperature dependent material. Consequently, the stress rates, $\dot{\boldsymbol{\sigma}}=\mathbf{C} \mathbf{D}^{e}$, can be solved by Eq.(3) and Eq.(6)

\subsection{Modified integration algorithm}

Elastic strain increments by strain space plasticity can be calculated analytically as shown in Eq.(7).

$$
\Delta \boldsymbol{\varepsilon}^{e}=\int_{t}^{t+\Delta t} \mathbf{D}^{e} d t=\mathbf{D} \Delta t-\frac{1}{2 \mu} \int_{t}^{t+\Delta t} d^{p l} \frac{\partial \varphi}{\partial \boldsymbol{\varepsilon}} d t
$$

Numerical integration of Eq.(7) can be carried out with 1st-order accuracy as follows,

$$
\Delta \boldsymbol{\varepsilon}^{e}=\int_{t}^{t+\Delta t} \mathbf{D}^{e} d t \approx \mathbf{D} \Delta t-\frac{1}{2 \mu} d^{p l} \Delta t \frac{\partial \varphi}{\partial \boldsymbol{\varepsilon}} .
$$

Eq.(8) shows the backward-Euler return mapping method which is the most widely used integration scheme. The backward-Euler return mapping method consists of elastic predictor-plastic corrector. The method has advantage that it is unnecessary to compute the intersection point of the elastic predictor with the yield surface when deformation occurs from inside the yield surface to plastic state. ${ }^{2}$ However the omission of the 2ndorder term in Eq.(8) leads to the truncated error and exact solution can be obtained only when the vector of plastic strain rate, $d^{p l} \mathbf{a}$, varies linearly during $t \leq \tau \leq t+\Delta t$.

Actually, the plastic deformation accompanies with nonlinearities and nonproportional loading. That is, $d^{p l} \mathbf{a}$ varies nonlinearly during the single time step. In order to consider these nonlinearities and non-proportional loading, the proposed algorithm integrates the 2nd term of Eq.(7) analytically using integration by part and chain rule as shown in Eq.(9).

$$
\Delta \boldsymbol{\varepsilon}^{e}=\mathbf{D} \Delta t-\frac{1}{2 \mu} \int d^{p l} \frac{\partial \varphi}{\partial \boldsymbol{\varepsilon}} d t=\mathbf{D} \Delta t-\frac{1}{2 \mu}\left[\int \frac{d}{d t}\left\{d^{p l} \Delta t \frac{\partial \varphi}{\partial \boldsymbol{\varepsilon}}\right\} d t-\int\left(d^{p l} \Delta t \frac{\partial^{2} \varphi}{\partial \boldsymbol{\varepsilon} \partial t}\right) d t\right]
$$

If the 2-stage Lobatto IIIA is applied to Eq.(9), It is possible to obtain the important results that the 2nd and 3rd term of R.H.S. in Eq.(9) become zero at time $\tau=t$ because of $d^{p l} \Delta t=\Delta \varepsilon^{p l}(t)=0$. Therefore it can be expressed only as the variables at time $\tau=t+\Delta t$, which means that it is unnecessary to compute the intersection point of the elastic predictor with the yield surface in the same manner of the backward-Euler return method. The results of numerical integration can be obtained as follows; 


$$
\Delta \boldsymbol{\varepsilon}^{e} \cong \mathbf{D} \Delta t-\frac{\Delta t}{2 \mu} d^{p l} \frac{\partial \varphi}{\partial \boldsymbol{\varepsilon}}+\frac{\Delta t^{2}}{4 \mu} d^{p l}\left(\frac{\partial^{2} \varphi}{\partial \boldsymbol{\varepsilon}^{2}}\left(\mathbf{D}-\frac{1}{2 \mu} \frac{\mathbf{a}^{T} \mathbf{D}}{3 \mu+H} \mathbf{a}\right)\right) .
$$

Adopting the proposed algorithm, the convective plastic strain can be considered since there is the 2nd derivative term of the yield function in the 3rd term in Eq.(10). Therefore the proposed algorithm enables to consider the histories of plastic strain during the plastic deformation and also has 2nd-order accuracy.

This study adopts the iteration procedure using the Newton-Raphson method to enforce consistency at the end of the step. ${ }^{2}$ The proposed algorithm is implemented with ABAQUS/Explicit using the user-subroutine, VUMAT. ${ }^{4}$

\section{Numerical Implementation}

\subsection{Accuracy assessment using the isoerror maps}

In order to evaluate the accuracy of the proposed scheme, isoerror maps are constructed using both the conventional and the proposed scheme. The isoerror maps which is proposed by Kreig et al. is popular method to assess the accuracy of the numerical integration algorithm for elasto-plastic problem. ${ }^{5}$ The isoerror maps are constructed using the three points on the yield surface, which correspond to uniaxial, biaxial and pure shear stress respectively. Errors are plotted as the relative root mean square of the error between the exact and computed solution, which is obtained according to the following expression.

$$
\delta=\frac{\sqrt{\left(\boldsymbol{\sigma}-\boldsymbol{\sigma}^{*}\right):\left(\boldsymbol{\sigma}-\boldsymbol{\sigma}^{*}\right)}}{\sqrt{\boldsymbol{\sigma}^{*}: \boldsymbol{\sigma}^{*}}} \times 100(\%) .
$$

, where $\sigma$ is the results from the proposed algorithm and $\sigma^{*}$ is the exact solution corresponding to the 1000 strain increments.
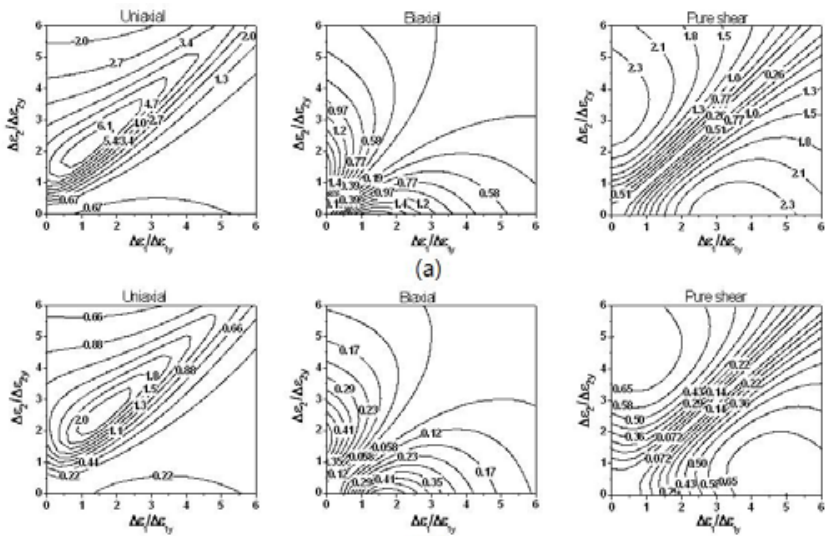

Fig. 1. Isoerror maps corresponding to uniaxial, biaxial and pure shear stress on the yield surface: (a) the conventional backward-Euler return method; (b) the modified integration algorithm. 
The accuracy assessment of this study adopts plane strain state and rate-independent Swift constitutive model shown in Eq.(12).

$$
g\left(\varepsilon^{p}\right)=832.85\left(0.0078+\varepsilon^{p}\right)^{0.182} M P a .
$$

Fig. 1(a) shows the isoerror maps of the conventional backward-Euler return method in Eq.(8) and Fig. 1(b) exhibits the results of the proposed algorithm. The isoerror maps indicate that the conventional backward-Euler return method gives the errors of 6.7, 1.9 and 2.6 on the uniaxial, biaxial and pure shear stress state respectively, while the proposed method provides the errors of $2.2,0.58$ and 0.72 . The comparison using the isoerror maps represents that the accuracy of the proposed algorithm is improved by $70 \%$ compared to the conventional backward-Euler return method at any given strain increment.

\subsection{Numerical analysis of adiabatic shear bands}

Numerical simulation of adiabatic shear band test performed by Kim et al. is carried out in order to demonstrate the accuracy of the proposed algorithm for dynamic deformation. Kim et al. used cylindrical shape of $93 \mathrm{~W}-4.9 \mathrm{Ni}-2.1 \mathrm{Fe}$ alloy as a specimen. The diameter and length of the specimen are $0.48 \mathrm{~mm}$ and $0.38 \mathrm{~mm}$ respectively. Fig. 2 represents the adiabatic shear band is localized in the specimen subjected to about $50 \%$ strain at 5000 /sec strain rate. ${ }^{6}$ Fig. 3 shows the finite element model of a specimen and incident, transmitted bars for the analysis of the adiabatic shear band. Finite element models utilize axi-symmetric elements for simplicity and infinite elements in order to prevent reflection of the stress waves on the ends of two bars. 5000 elements are used in the modeling of the specimen as shown in Fig. 3.

In the simulation, the Johnson-Cook model is used as the rate and temperature dependent constitutive relation as shown Eq.(12). The values of material constants are taken from Stevens's report. ${ }^{7}$

$$
\begin{aligned}
& \sigma_{y}=\left(A+B \varepsilon^{p^{n}}\right)\left(1+C \ln \left(\dot{\varepsilon}^{p} / \dot{\varepsilon}_{o}\right)\left(1-T^{*}\right)\right. \\
& \text { where, } \\
& \mathrm{A}=1506 \mathrm{MPa}, \mathrm{B}=177 \mathrm{MPa}, \mathrm{C}=0.016, \mathrm{n}=0.12, \mathrm{~m}=1.0, \dot{\varepsilon}_{\mathrm{o}}=1 / \mathrm{s}
\end{aligned}
$$

Distributed load is imposed on the end of the incident bar shown in Eq.(14) so that the specimen is subjected to about $50 \%$ strain at $5000 / \mathrm{sec}$ strain rate.

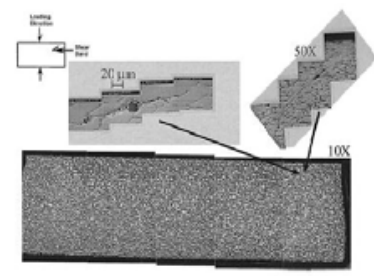

Fig. 2. Adiabatic shear bands of W-Fe-Ni alloy.

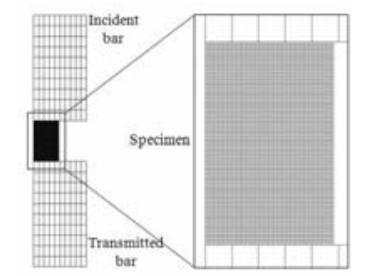

Fig. 3. Finite element model for formation of the adiabatic shear band. 

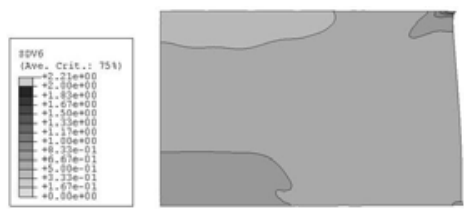

(a)

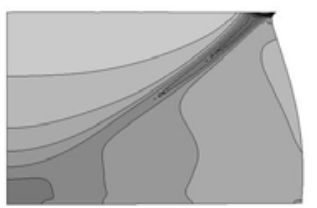

(b)

Fig. 4. Deformed shapes and contours of the effective plastic strain: (a) ABAQUS/Explicit; (b) ABAQUS/Explicit VUMAT.

$$
P=\left\{\begin{array}{c}
10 \mathrm{kN} / \mathrm{mm} \text { if } 0<\mathrm{t}<100 \mu \mathrm{s} \\
0 \quad \text { if } 100<\mathrm{t}<150 \mu \mathrm{s}
\end{array}\right.
$$

Fig. 4(a) shows the deformed shape and contour of the distribution of effective plastic strain obtained from ABAQUS/Explicit, while Fig.4(b) represents the ones obtained from ABAQUS/Explicit VUMAT. Homogeneous deformation is observed throughout the 50\% strained specimen as shown in Fig. 4(a). Compared with the results of the conventional scheme, the suggested scheme indicates that the adiabatic shear bands are formed in the specimen and propagated to the center of the bottom from the edge of the specimen as shown in Fig. 4(b). Comparing the results from ABAQUS/Explicit and ABAQUS/Explicit VUMAT, the proposed algorithm gives a better agreement with Kim's experimental results.

\section{Conclusions}

This paper is concerned with modified integration algorithm on the strain space for rate and temperature dependent elasto-plastic constitutive relations in order to obtain more accurate results in numerical implementation. The proposed algorithm is integrated analytically using integration by part and chain rule and then is applied to the 2-stage Lobatto IIIA with second-order accuracy. Accuracy assessment using the isoerror maps indicates the result of the proposed algorithm is improved by $70 \%$ compared to the conventional backward-Euler return method. Numerical analysis of adiabatic shear band also demonstrates that the proposed algorithm is more accurate than the conventional method for rate and temperature dependent constitutive model.

\section{References}

1. A. S. Khan, S. Huang, Continuum Theory of Plasticity (John Wiley \& Sons, ngland, 1995).

2. M. A. Crisfield, Non-linear Finite Element Analysis of Solids and Structures. Volume I (John Wiley \& Sons, England, 1991).

3. J J. D. Lambert, Numerical Methods for Ordinary Differential Systems (John Wiley \& Sons, England, 1991).

4. Hibbit, Karlsson and Sorensen, ABAQUS/Explicit User's Manual (Hibbit, Karlsson \& Sorensen Inc., Pawtucket. Rhode Island, 2004).

5. J. C. Simo, T. J. R. Hughes, Computational Inelasticity (Springer, New York, 1998).

6. D. S. Kim, S. Nemat-Nasser, J. B. Isaacs and D. Lischer, Mech. Mater. 28, 227 (1998)

7. J. B. Stevens, MS Thesis, Virginia Polytechnic Institute and State Univ., 1996 